\title{
Corela
}

Cognition, représentation, langage

HS-27 | 2019

Co(n)textualisation(s)

\section{De l'entorse à la norme à l'atypicité typique : Retour sur un corpus de SMS de sourds et ses métadonnées}

\section{Michel Otell}

\section{(2) OpenEdition}

Journals

Édition électronique

URL : http://journals.openedition.org/corela/7831

DOI : $10.4000 /$ corela.7831

ISSN : 1638-573X

Éditeur

Cercle linguistique du Centre et de l'Ouest - CerLICO

Référence électronique

Michel Otell, « De l'entorse à la norme à l'atypicité typique : Retour sur un corpus de SMS de sourds et ses métadonnées », Corela [En ligne], HS-27 | 2019, mis en ligne le 12 avril 2019, consulté le 20 avril 2019. URL : http://journals.openedition.org/corela/7831; DOI : 10.4000/corela.7831

Ce document a été généré automatiquement le 20 avril 2019.

\section{(c) (i) (2)(2)}

Corela - cognition, représentation, langage est mis à disposition selon les termes de la licence Creative Commons Attribution - Pas d'Utilisation Commerciale - Partage dans les Mêmes Conditions 4.0 International. 


\title{
De l'entorse à la norme à l'atypicité typique : Retour sur un corpus de SMS de sourds et ses métadonnées
}

\author{
Michel Otell
}

1 L'ingénierie d'une méthodologie de recueil de données est forcément motivée par la problématisation d'une série d'observations relevées par l'analyste. En sens inverse, c'est le regard situé de l'observateur, analyste, chercheur, qui, après une phase de recueil d'expériences éparses et diverses sur le terrain, laisse émerger chez lui des questionnements qui donnent lieu à la formulation d'une problématique de recherche, qui, elle, dirigera et contraindra l'élaboration du protocole de recueil des données. Autrement dit, la problématique n'est pas donnée par le terrain; c'est l'expérience du terrain qui, mise à l'épreuve du positionnement - épistémologique, culturel, subjectif - de l'analyste, laisse naître chez lui une problématisation. Or le protocole de recueil des données de terrain est souvent conçu dans une tension avec les contraintes et les potentialités fixées par la problématique. En conséquence, même dans le cas où il est constitué de données discursives naturelles et spontanées, c'est-à-dire de productions discursives qui ne sont pas induites par l'activité de recherche, ni créées par le chercheur, un corpus et ses métadonnées trahissent toujours un positionnement épistémologique, et donc culturel et subjectif. Naturellement, il serait inutile de se lancer dans la collecte d'un corpus dit naturel et spontané dans l'espoir de se prémunir de biais méthodologiques, d'ethnocentrisme, ou d'éventuelles déformations épistémologiques. Le choix de travailler sur des données de terrain, de les enrichir de métadonnées, de limiter autant que possible les interventions de l'analyste sur la matière discursive, ne permet pas de faire l'économie d'un recul critique sur son positionnement de chercheur, mais nous invite, au contraire, à porter sur nous-même un regard sans concession. Plus encore, le corpus naturel est l'occasion de formaliser à chaque étape et à même la trame du travail de recherche, les fondements épistémologiques, culturels, et subjectifs de notre positionnement.

2 Nous proposons ici d'observer le cas d'un corpus de SMS de personnes sourdes, et ses métadonnées. Le but est de porter un regard rétrospectif notamment sur les motifs de son 
cadrage, l'enquête de terrain préalable à sa collecte, le protocole de collecte en tant que telle, le défi que constitue son annotation, et ceci dans le but de discuter les avantages et les limites de la méthodologie retenue au vu des particularités des données recueillies.

3 Nous présenterons d'abord le corpus en question tel qu'il se trouve dans sa forme finale, avec ses métadonnées (1.1.). Ensuite, nous expliciterons sa genèse, depuis le questionnement qui en est à l'origine, jusqu'aux compromis qui ont permis sa finalisation, en mettant autant que possible ce processus en rapport avec la réalité sociolinguistique des sourds aujourd'hui en France (1.2.). Ceci permettra de présenter de façon informée un échantillon choisi de données discursives issues du corpus, qui sera analysé dans une visée critique (1.3.), dans l'optique d'offrir un regard rétrospectif critique sur la réalisation du corpus (2.). En conclusion, l'expérience de la collecte du corpus sera mise en perspective avec les contraintes et les apports du terrain de l'enquête (3.).

\section{Méthodologie : un corpus de SMS conversationnels entre sourds signants}

\subsection{Présentation du corpus et de ses métadonnées}

Il s'agit d'un corpus constitué de conversations par SMS (Short Message Service (Wray, 2002 in Tagg, 2012:1)). Chaque conversation représente un fil d'échanges de messages texte entre deux utilisateurs de téléphone portable, comprenant la totalité des messages échangés contenus dans le téléphone du participant donneur au moment de l'extraction. Ce fil d'échanges est la compilation de tous les messages envoyés et de tous les messages reçus, dans l'ordre d'enregistrement de ces opérations, dans le téléphone portable du participant donneur. C'est-à-dire que si, par exemple, une défaillance du réseau téléphonique avait retardé la réception ou l'émission par le téléphone du participant donneur de quelques SMS, ce retard étant visible par l'utilisateur dans le déroulement de l'échange tel qu'il s'affiche à l'écran, il sera également visible dans le corpus. Par ailleurs, chaque fil est par nature constitué d'une succession d'échanges conversationnels de taille très variable. Il peut s'agir d'une question restée sans réponse (« Laisse ton portable et iPad dans ma chambre .. [PRÉNOM] $]^{1}$ est avec toi ? » envoyé un matin, puis « Ça va ? Tu es a la maison? » envoyé l'après-midi suivant), comme d'une longue conversation s'étendant sur plus d'une journée, et entrecoupée d'interruptions de longueurs variables. Ces échanges sont tous dialogaux. Au total, le corpus se constitue de cent quatre-vingt-seize fils d'échanges, comptant chacun entre quatre et deux cent soixante SMS individuels, pour un total de deux cent quatre-vingt-dix donneurs. Il a été récolté sur une période de plus de deux ans, et mis en forme pendant près d'un an.

Chaque fil d'échange a fait l'objet d'un don par un des deux participant, et avec l'accord écrit des deux. L'opération s'est faite à l'aide d'un logiciel commercial initialement conçu pour conserver dans un ordinateur des éléments choisis du contenu d'un téléphone. Chaque fil d'échanges est disponible en trois formats différents, dont un format image se rapprochant de l'affichage par défaut de l'écran du téléphone du participant donneur. Le corpus rend compte, pour chaque message texte pris individuellement, de s'il a été émis ou reçu, ainsi que de la date et de l'heure de l'enregistrement de l'opération d'émission ou de réception dans le téléphone. Il fait également apparaître la grande majorité des symboles, émoticônes et autres binettes employés. 
6 Lors de l'opération de récolte du fil d'échange, chacun des participants a fait l'objet d'un entretien semi-directif dont le produit participe des métadonnées du corpus de SMS. L'entretien a porté sur le parcours linguistique de chaque participant et de sa famille, sur son parcours scolaire et de formation, sur son usage du téléphone portable, et sur sa relation avec son interlocuteur.

7 Le corpus est ainsi structuré qu'à partir des données brutes - la matière discursive prise dans son environnement iconographique partiellement reconstitué -, se déploient une première strate de métadonnées (les heures et dates d'enregistrement des opérations d'émission et de réception de chaque SMS pris individuellement, la trace des envois de fichiers variés (les photographies et autres vidéos n'apparaissent pas dans le corpus, mais apparait la trace de leur transmission ou de leur réception ${ }^{2}$ ), puis une seconde strate de métadonnées issue des entretiens.

\subsection{Contexte, cotexte, indices de contextualisation, données, métadonnées : la méthodologie de collecte du corpus}

8 Comme dans toutes les études des écrits de personnes sourdes, l'hypothèse générale qui a appelé la constitution de notre corpus prend sa source dans une observation largement répandue dans la littérature scientifique comme dans les institutions scolaires accueillant des personnes sourdes: les productions écrites des sourds présenteraient souvent des atypicités si singulièrement spécifiques qu'elles sembleraient permettre au lecteur aguerri de reconnaître qu'elles ont été écrites par des sourds. Si la littérature propose des lectures variées de ces atypicités, allant du langage pathologique au sociolecte sourd, ou au lecte d'apprenant, c'est généralement dans un travail de recensement des écarts à la norme que s'étayent les méthodologies. Nous ne proposions pas alors d'effectuer un travail dont l'ambition en bout de ligne aurait été de participer à l'amélioration des méthodes d'enseignement du français au public sourd, mais d'éclairer nos modélisations du langage en les mettant à l'épreuve de ces productions atypiques.

La question d'origine était d'exploiter la notion de production du sens telle qu'elle est définie par la praxématique et d'autres linguistiques cognitives, énonciatives ou anthropologiques (Détrie et al, 2017). Il s'agissait d'étudier le français écrit par des sourds signants et d'y chercher d'éventuelles interférences entre LSF et français. Le terme d'interférence est souvent employé par les linguistiques des contacts de langues pour désigner les phénomènes de mélanges, transferts et emprunts d'une langue à une autre. Ceci s'applique à la syntaxe, à la grammaire, au lexique, ainsi qu'aux compétences et usages conversationnels ou communicationnels (politesse, déférence, excuses, enchaînement des tours de parole, etc.). L'objet de l'étude n'était pas de faire émerger les influences morphosyntaxiques entre les deux langues, bien que cela soit une étape nécessaire de l'analyse. La question portait plutôt sur les éventuels phénomènes de transfert entre les schémas praxiques de la LSF et ceux du français à l'écrit. La praxématique appelle schéma praxique un ensemble de représentations du monde et de rapports qu'entretient un sujet avec une réalité qu'il envisage en discours à l'aide de ses expériences (« une structure praxique préexistante [...] qui permet la conceptualisation de la notion à représenter, en imposant ses propres réseaux sémantiques, et en fournissant un cadre expérientiel qui met en valeur un aspect de la réalité à signifier. " (Détrie, 2002: 84). Ces schémas socio-expérientiels sont à rapprocher des fondements des métaphores ontologiques chez Lakoff \& Johnson (2011), par exemple. 

pas comme des écarts avec une norme standardisée du français, mais résolument comme les marqueurs d'une créativité linguistique qui, en se déployant nécessairement à l'intérieur des limites de l'opérationnel - du compréhensible -, révèlent des opérations cognitives se déroulant généralement à l'ombre de la correction grammaticale, en bon français.

11 La particularité de ce corpus réside donc en ce qu'au moins l'un des deux participants de chacun des fils conversationnels est sourd et locuteur de la Langue des Signes Française. Dans les faits, il a été impossible de nous limiter aux seules personnes présentant une surdité profonde de naissance ou précoce, et un bilinguisme LSF-français écrit, et non oralisants (c'est-à-dire qui n'utilisent pas la prononciation vocale du français - avec ou sans rétrocontrôle auditif - comme mode de communication majeur au quotidien), pour la constitution de la cohorte de donneurs sourds. Cette impossibilité vient de la réalité sociologique - et donc historique - des sourds en France aujourd'hui, qui n'est pas directement l'objet du présent travail, bien qu'elle le contraigne très fortement. Si les premiers contacts avec les donneurs sourds comme entendants se sont souvent fait par SMS et en français, les entretiens avec les donneurs sourds ont tous été menés en LSF, et ceux avec les donneurs entendants ont été menés en français ${ }^{3}$. Il faut enfin préciser que la LSF ne peut être écrite de manière aisée par ses locuteurs (Boutet \& Garcia, 2003), ce qui explique que tous les SMS du corpus sont en français, bien qu'ils présentent, nous le verrons, des atypicités qui les différencient singulièrement des SMS échangés entre francophones entendants (Panckhurst, et al, 2016) La figure 1 donne un aperçu du corpus dans son format image. Pour des raisons
d'anonymisation, nous n'avons pas fait apparaître les dates, heures, et identifiants des
participants.

\section{Je suis pressé chez moi pour mon lit mais encore $2 \mathrm{~h}$}

Oui patience encore $1 \mathrm{~h}$

\section{Qui ma voiture non merci est mieux train}

\section{Eh oui je comprends .. Mieux avion 1 h non ?}

\section{Tu m offerte pour avion}

Hohihoh bonne nuit je vais me coucher et Bo courage pour demain

Merci Bo nuit et bo vacances ma coeur ९०

\section{Problème réseau pas grave je pense bien à toi}



d'années, dans une conversation avec son compagnon. Les deux interlocuteurs sont sourds

profonds de naissance. La participante en bleu a été appareillée dans sa prime enfance (il ne s'agissait pas d'un implant cochléaire, mais d'un appareil plus répandu qui permet d'amplifier certaines longueurs d'ondes davantage que d'autres afin de fournir à l'oreille un signal sonore plus adapté à ses traits d'audition). Elle a ensuite suivi une éducation strictement oralise jusqu'à ses vingt ans. C'est-à-dire, dans ce cas, qu'elle a été scolarisée en milieu dit ordinaire, accompagnée d'Assistants de Vie Scolaire (AVS), à l'école, puis à l'Université, où elle a également pu faire appel aux services de preneurs de note. Elle a également suivi des consultations hebdomadaires avec une orthophoniste depuis sa prime enfance et jusqu'à ses vingt ans. Il est intéressant de noter que, de tous les donneurs sourds de notre cohorte, elle est la seule à déclarer revoir régulièrement son ancienne orthophoniste malgré la fin des consultations, et même avoir une relation suffisamment proche avec elle pour la compter parmi ses amis au sens élargi. Tous les autres sujets sourds de la cohorte déclarent avoir subi leurs relations avec les orthophonistes, et les dépeignent très négativement. La participante en question a rencontré la LSF dans la vingtaine. Elle a un diplôme universitaire, et un emploi qui la met au contact de personnes sourdes. Ses compétences linguistiques et culturelles en français, autrement dit, son niveau de français, est parfaitement ordinaire, et ses compétences en LSF sont très bonnes ${ }^{4}$.

C'est son compagnon qui est le scripteur gris dans la figure 1. Bien qu'on repère des particularités dans les écrits en bleu et en gris, c'est lui qui a un français plus hésitant. Son parcours est différent de celui de sa compagne. Comme cette dernière le déclare ellemême dans les entretiens, elle "s'adapte pour se faire comprendre ». Notre participant don le discours est en gris est décrit par sa compagne comme étant « sourd signant à 100 \% ». Dans les entretiens, il apparaît qu'il n'oralise presque jamais, et considère lui-même qu'il n'écrit pas en «bon français ». Il a été appareillé dans la prime adolescence pendant un peu plus d'un an, mais l'expérience a échoué à cause, notamment, de douleurs. Enfant, il communiquait en mimant, et sa relation avec sa famille (ses parents n'ont appris quelques rudiments de LSF que très tardivement) n'a pas été très riche, selon l'image qu'il en donne. Il a un Brevet d'Études Professionnelles (BEP), et un emploi qui l'amène à se déplacer très fréquemment. Ceci favorise l'usage des SMS pour communiquer avec sa compagne.

Naturellement, en plus des saillances que l'on observe déjà à la lecture de l'extrait présenté dans la figure 1, la matière discursive d'un tel corpus présente plusieurs autres caractéristiques qui se télescopent : d'une part, la situation n'énonciation ${ }^{5}$ présente deux ego, les deux participants à l'échange; d'autre part, elle compte deux hic, puisque les participants échangent à distance et en absence; mais le nunc peut être soit unique, si les interlocuteurs sont disponibles et acceptent de participer à une conversation en direct - comme un tchat-, soit le nunc peut être multiple si l'un des participants n'est pas immédiatement disponible, et ceci peut arriver d'emblée, ou à tout moment au cours de la conversation.

17 Par ailleurs, le cadre de l'interaction ${ }^{6}$ diffère évidemment d'une paire de locuteurs à l'autre, avec la variété des relations entretenues et de leurs enjeux (parent/enfant, époux,

Corela, HS-27 | 2019 
amis, collègues, etc.) ; mais le cadre de l'interaction peut également varier selon l'activité en cours (faire les courses, rentrer de voyage, organiser une activité, se faire la cour, ...).

Enfin, il ne faut pas oublier le téléphone portable (ici, toujours un smartphone $e^{7}$ ), qui contraint également la matière discursive dans sa forme par son ergonomie (on pense à la correction automatique intempestive ou non, aux majuscules automatiques, aux émoticônes, photos, vidéos, à la géolocalisation, à la petitesse des touches tactiles, aux problèmes de connexion, à l'usage économique de l'abréviation ou du style télégraphique, à la licence de ne pas corriger les coquilles ou erreurs jugées mineures, ou à la possibilité de passer de l'écriture à la visiophonie pour signer en LSF et communiquer de façon parfois plus fluide).

Tout ceci est évidemment nécessaire à l'analyse du discours dans le corpus, et gagne à être mis en face des données recueillies à l'aide des entretiens semi-directifs avec les donneurs des SMS. Reste que les saillances que la figure 1 donne à voir résistent à l'observation à cette échelle.

\subsection{Des saillances aux atypicités typiques : observation d'un échantillon du corpus}

La figure 2 présente un extrait du même fil d'échanges, et qui se situe à l'initial d'une interaction plus longue. L'activité en cours est la suivante (telle que reconstituée à l'aide des indices de contextualisation relevés dans l'échange) : le scripteur en gris est en train de faire des courses sous une pluie forte, et donne de ses nouvelles à sa compagne en bleu. Sur la période pendant laquelle se déroule l'interaction, le couple ne loge pas au même endroit.

Je vais la course que rien

Ici voir rien par la pluie tres fort

Oh pauvre !!

Ici partout la grêle jamais vu mais fin la course pour tranquiller 

jamais vu mais fin la course pour tranquiller ». Rappelons que nos métadonnées nous apprennent que les deux interlocuteurs sont sourds profonds de naissance, et signants ; qu'ils utilisent généralement la LSF pour communiquer entre eux; et que la participante en bleu, qui a par ailleurs un excellent niveau de français, a généralement le sentiment d'adapter son parler pour se faire comprendre de son compagnon, qui juge ne pas avoir un «bon niveau de français ». Il apparaît alors, à la vue des saillances du tour de parole étudié, qu'il n'est pas inutile de tenter une transposition du tour en LSF pour tenter d'en faire l'exégèse. Nous utiliserons pour cela une convention de transcription commode qui est très répandue parmi les locuteurs de la LSF pour la noter à l'écrit. Précisons que bien que d'usage facile, cette convention n'est pas suffisante à la bonne écriture de la LSF, ne serait-ce que parce qu'elle ne permet pas de représenter les mimiques faciales, les positions corporelles, le rythme de signation, l'activité oculaire du locuteur, ou encore d'autres particularités grammaticales et syntaxiques de la LSF telles que les transferts situationnels, les transferts personnels, et les proformes (Cuxac, 2000), par exemple, qui ne sont pas directement l'objet du présent travail.

Proposition de transposition du français à la LSF :

«Ici partout la grêle jamais vu mais fin la course pour tranquiller »

[partout] [grêle] [grêle] [grêle] ${ }^{8}$ [jamais vu]

- parataxe- ${ }^{9}$ [caddie] [fini]

-parataxe- [calme]

Proposition de retransposition de la LSF au français :

$\rightarrow$ Il grêle très très fort, je n'ai jamais vu ça! Je vais quand même faire des courses

pour être tranquille.

Dans ce tour, plusieurs phénomènes sont saillants. Nous nous proposons de les observer linéairement selon l'ordre syntaxique du tour original.

D'abord l'adverbe «ici», déictique (locatif), est en réalité actualisé dans un usage qui n'est pas aussi ordinaire qu'il peut le sembler à première lecture. Il est en usage déictique total, c'est-à-dire qu'il saisit tout à la fois ego, hic, et nunc. Le locuteur, en écrivant « ici ", dit en fait «moi, qui me trouve ici, en ce moment, j'observe depuis ma situation, et te relate mes observations». C'est un usage fréquent dans le corpus, mais uniquement attribuable aux scripteurs sourds. Ailleurs dans ce même fil d'échanges, nous pouvons voir la compagne de notre scripteur écrire « Moi contente de te voir !! Ici arrive odysseum ${ }^{10}$ ", alors qu'elle est en train de décrire sa route en temps réel. Notons tout de même l'absence du signe [ici] dans notre proposition de transposition du français à la LSF. Si le signe standard [ici] existe bien en LSF, notre expérience empirique de cette langue en situations naturelles semble montrer qu'il serait incongru à l'initial de ce tour de parole. La LSF, comme toutes les langues signées naturelles, est une langue de la coprésence. Paradoxalement, elles sont en quelque sorte des langues de l'oralité, dans la mesure où elles ont émergé de la socialisation des sourds, qui, avant l'ère toute récente de la visiophonie, n'était possible qu'en situation de coprésence. Ces langues ne s'écrivent pas. Elles ne se laissent pas représenter dans la pierre, l'argile, ou le parchemin. Avant l'apparition de la vidéo, il n'y avait jamais eu de situation où un locuteur d'une langue signée aurait pu fixer matériellement son discours pour le transmettre, après un délai de temps, à un interlocuteur absent lors de la profération. L'ici et le maintenant étaient uniques et strictement associés. La difficulté de représenter matériellement la LSF

Corela, HS-27 | 2019 
- autrement que par la vidéo - se voit nécessairement éclairée par la primauté de la coprésence dans les langues signées. Elles sont faites de telle façon qu'il faut un corps humain pour les actualiser, et qu'il faut donc voir ce corps humain pour être allocutaire. Pensons notamment aux avatars numériques, humanoïdes, et signants, que les voyageurs peuvent apercevoir sur des écrans de télévision dans certaines gares, et qui signent des annonces aux usagers sourds des trains. Disparu dans le travail de transposition du français à la LSF, ce déictique total aurait pu réapparaitre dans la retransposition en français, peut-être sous la forme d'un présentatif : « Il y a beaucoup beaucoup de grêle ». Ceci dit, la construction impersonnelle «il grêle » nous semble ici suffisante à rendre le sens du tour.

Attachons-nous maintenant à étudier la locution «jamais vu». Cousine de «c'est du jamais vu », en français, il semble qu'il s'agisse en réalité d'une transposition directe de la LSF au français. Il existe en effet en LSF un signe standard noté [jamais vu], et très répandu. Sur le plan morphologique, il s'agit d'une contraction de [jamais] et de [vu], et qui se profère gestuellement généralement accompagnée de ce qu'on appelle une labialisation ${ }^{11}$, c'est-à-dire une articulation (buccale) silencieuse des mots français « jamais vu ». En LSF, , [jamais vu] n'a pas seulement le sens de « je n'ai jamais vu X », mais également le sens plus exclamatif, plus expressif, de « c'est du jamais vu !, c'est inouï ! ». Il faut noter que malgré la structure interne de la profération buccale silencieuse «jamais vu», le signe, dans la syntaxe de la LSF, ne se conjugue, ni ne s'accorde, ni même n'entretien les relations sémantico-syntaxiques attendues en français. Par ailleurs, le signe [jamais vu] est directionnel. C'est-à-dire qu'il doit être proféré de telle sorte qu'il pointe vers l'objet inouï, ou vers le lieu de sa représentation dans le volume ${ }^{12}$ de signation.

Pour ce qui est de "fin la course », il est préférable d'élargir la focale, et de considérer l'entoure lexicale: «mais fin la course pour tranquiller». Nous avons proposé la transposition «Je vais quand même faire des courses pour être tranquille. ». En effet, il semble qu'il faille entendre ici par "fin la course» un équivalent de "à la fin des courses ", ou " une fois les courses faites ». On remarque l'usage de course au singulier au lieu du pluriel attendu. On note également l'usage atypique de fin, qui n'est pas ici un substantif. Nous y voyons en fait un usage prépositionnel si on fait l'analogie avec « après les courses »; ou alors un usage qui pourrait être qualifié de participial absolu, sur le modèle de l'ablatif absolu du latin: «les courses finies, je serai plus serein». La transposition du français à la LSF permet d'y gagner en clarté : [caddie] [fini] -parataxe[calme]. Le signe [caddie], qui reprend icôniquement l'activité de pousser un caddie dans un supermarché, actualise ici un équivalent de «faire ses courses $»^{13}$. Le signe [fini] a un usage en LSF qui le rapproche d'une particule verbale aspectuelle. C'est-à-dire que, proféré conjointement à un verbe, le signe [fini] opère un réglage du sens véhiculé par ledit verbe en lui conférant un aspect accompli. Ce mécanisme est à rapprocher par exemple de la composition des temps du français (je mange $\rightarrow$ j'ai mangé ; je mangeais $\rightarrow$ j'avais mangé ; je mangerai $\rightarrow$ j'aurai mangé) qui actualise l'aspect accompli. La LSF dira «[caddie] [fini] » pour signifier ce que le français dirait « une fois les courses faites », ou "quand j'aurai terminé les courses ». Notons encore que le signe [fini] se profère avec la labialisation « fini », de la même façon qu'exposé plus haut au sujet du signe [jamais vu].

Attardons-nous maintenant sur le dernier mot du tour de parole : «tranquiller ». Nous y voyons une construction très riche. Il ne s'agit pas, à nos yeux, d'une simple transposition de la LSF au français. Nous y voyons un fait de créativité. La proposition de transposition 
en LSF traduit «tranquiller » ainsi : [calme], dans une proposition parataxique. Ce signe se profère avec une mimique de sérénité. Morphologiquement, le signe [calme] peut fonctionner comme un verbe, comme un substantif, ou comme un adjectif, sans modification significative ${ }^{14}$, si ce n'est qu'il peut être directionnel (pour dire «calme-toi ", il suffit d'effectuer le signe dans la zone du volume de signation la plus proche de l'interlocuteur, en appuyant un contact visuel; pour dire «je me calme», il suffit d'effectuer le signe directement sur sa propre cage thoracique, en regardant vers le ciel par exemple). Le signe [calme] est un signe mobile (les mains changent de configuration, et légèrement d'emplacement, au courant de la signation), et qui mobilise la face du locuteur (par la mimique de sérénité et les contacts visuels éventuels), de telle sorte qu'il est obligé d'incarner corporellement la sérénité et le calme, dans une coénonciation radicalement visuelle. Se calmer, trouver la sérénité, peut être envisagé comme un verbe d'action en LSF. C'est bien cela que notre locuteur sourd en gris (figure 2) a voulu actualiser en français, dans le cadre morphosyntaxique du français, en écrivant «tranquiller », contre, certainement, les résistances de son correcteur automatique. Dans la forme morphologiquement verbale "tranquiller ", le locuteur laisse apparaître non seulement son bilinguisme LSF-français écrit, mais surtout sa biculturalité visuo-gestuelle et écrite.

Rappel de notre proposition de transposition du français à la LSF, puis de la LSF vers le français :

" Ici partout la grêle jamais vu mais fin la course pour tranquiller »

[partout] [grêle] [grêle] [grêle] [jamais vu]

-parataxe- [caddie] [fini]

-parataxe- [calme]

$\rightarrow$ Il grêle très très fort, je n'ai jamais vu ça! Je vais quand même faire des courses

pour être tranquille.

Cet extrait choisi montre l'utilité d'effectuer un aller-retour du français à la LSF, et de la LSF au français. Cet exercice permet d'incarner le sens, en le représentant, en le mimant, en l'incorporant, jusque dans ses mimiques faciales et son regard. C'est une mise en scène. En y regardant de plus près, on devine une reconstitution de la chronologie des expériences intimes du locuteur. $1^{\circ}$ La sidération devant une tempête de grêle exceptionnelle, $2^{\circ}$ mener les courses jusqu'à leur terme, $3^{\circ}$ trouver la tranquillité. On ne retrouve pas cet effet dans l'équivalent en français « je finis les courses malgré la grêle pour être tranquille». Une telle construction ne serait pas choisie par un locuteur sourd de la LSF, qui préférerait l'ordre chronologique des expériences : la grêle, les courses, la tranquillité. Par le choix de "tranquiller », le locuteur prend l'interlocuteur par la main, et l'invite, le force même, à vivre la situation comme s'il était dans sa peau. Le locuteur fait émerger chez sa compagne des expériences $1^{\circ}$ de sidération, $2^{\circ}$ d'effort, $3^{\circ}$ de précaution, $4^{\circ}$ de soulagement, et $5^{\circ}$ de sérénité. C'est un formidable procédé argumentatif, en quelque sorte, de partage de subjectivité. C'est une manière d'imposer sa propre subjectivité à l'allocutaire, pour nourrir sa pulsion communicationnelle (Détrie et al., 2017). 


\section{Discussion}

31 Ces phénomènes de transposition par les locuteurs de la LSF au français pour « jamais $\mathrm{vu}$ », ces usages grammaticaux atypiques pour «fin la course», ces faits de créativité comme «tranquiller », ou ces constructions syntaxiques corporalisées et chronologisées, comme d'autres phénomènes que nous n'avons pas l'espace de présenter ici, sont très largement récurrents dans le corpus. Plus encore, même s'ils se retrouvent préférentiellement chez les locuteurs sourds, ils sont aussi parfois produits par certains scripteurs entendants quand ils s'adressent à un allocutaire sourd. Nous rencontrons aussi, dans ce corpus, des locuteurs sourds qui emploieront ces atypicités avec certains autres sourds, mais pas avec d'autres, ou avec des entendants. Ceci montre que les sourds bilingues ont conscience de l'existence de ce qui s'apparente à un lecte (Perini, 2013), ou à un parler, un usage, une collection d'usages. C'est précisément à la récurrence de ces saillances dans le discours écrit naturel et spontané des sourds signants de notre corpus, que nous faisons référence dans notre usage du terme atypicités typiques.

Un SMS comme «Ici partout la grêle jamais vu mais fin la course pour tranquiller » reste une curiosité même si les métadonnées recueillies lors de la collecte du corpus permettent de retracer le parcours linguistique et social du scripteur (sourd) et de son interlocuteur. Plus encore, le fait que de telles atypicités ne soient pas des hapax, qu'elles ne soient pas le fait d'un seul locuteur, ou d'une poignée, mais qu'elles se retrouvent régulièrement dans une majorité du corpus, installe, en quelque sorte, une typicité des atypicités. La mise en relation $1^{\circ}$ des caractéristiques dues à la forme SMS, avec $2^{\circ}$ les caractéristiques issues des métadonnées sur les locuteurs, et $3^{\circ}$ une classification lexicosémantique des phénomènes saillants en question, montre certes que, si les écarts à la norme du français standard qui ont déjà été observés dans d'autres études de SMS d'entendants [citer 88mil] se retrouvent également dans les SMS de personnes sourdes, les échanges comprenant au moins un participant sourd présentent des écarts d'un tout autre degré. En revanche, pour proposer une description adéquate de ces atypicités, pour expliquer leur apparition dans l'écrit en français de personnes sourdes, pour reconstituer l'intention du locuteur, il semble nécessaire de saisir chaque forme dans ses dimensions idiosyncrasique, pragmatique, relationnelle, et sociale.

Face à une population largement exclue de la littératie, largement exclue de la société des entendants, largement traversée par des rapports conflictuels et frustrants à leur langue, à notre langue, et à leur identité, c'est une approche totale, immersive, pour laquelle nous avons dû opter (Holcomb, 2013 ; Delaporte, 2002 et 2007 ; Mottez, 1999 et 2006).

La configuration de la communauté sociolinguistique que constituent les sourds signants français, et les particularités culturelles et anthropologiques qui la sous-tendent ont fortement contraint l'activité de collecte des données. Dans l'optique de mener à bien une analyse sémantique compréhensive, il a fallu apprendre la LSF, fréquenter la communauté des sourds signants de Montpellier, assister à des événements culturels, participer à des événements politiques, militants, et se familiariser avec le quotidien des sourds en France. Les premières tentatives d'obtention des SMS ont été un échec. Longtemps, les sourds que nous approchions promettaient de faire don de leurs SMS, mais ces promesses restaient toujours sans suite. Il a fallu une longue période d'approche, avant de recevoir la confiance de suffisamment de membres de la communauté locale des sourds signants. C'est seulement ensuite que nous avons obtenu, petit à petit, certains des 
SMS promis. Et nous remercions infiniment les sourds que nous avons rencontrés tout au long de ce travail, et spécialement ceux qui ont joué un rôle d'informateurs, selon les usages des approches anthropologiques, qui nous ont été d'un grand secours à de nombreuses reprises.

\section{Conclusion}

Le cas de ce corpus de SMS conversationnels entre sourds signants est un exemple de l'importance d'une étape préalable à la conception-même de la méthodologie de collecte des données sur le terrain. Cette étape préalable, quand elle consiste en une immersion de l'analyste dans le terrain où évolue la communauté linguistique étudiée, peut permettre de rendre plus efficaces les efforts de désethnocentration du chercheur. Mené en amont de tout recueil de corpus, un travail attentif de recensement, d'analyse, et de mise en perspective des enjeux sociaux, économiques, historiques, identitaires, politiques, et épistémologiques, du terrain d'étude, permet d'élaborer une méthodologie adaptée de recueil des données. C'est l'élaboration de la méthodologie de recueil que nous avons appelée ici l'ingénierie de la collecte du corpus. Elle comprend bien sûr la conception de la collecte des données (ici discursives) elles-mêmes, mais elle comprend également, et dès l'amont du travail, l'identification des différentes métadonnées de tout ordre qui seront utiles à une analyse informée des données du corpus. Dans une configuration aussi complexe que celle du bilinguisme-biculturalisme des sourds profonds de naissances qui naviguent entre LSF et français écrit, il apparaît essentiel de ne pas envisager le contexte de production de la matière discursive étudiée comme une ressource disponible a posteriori. Il semble réducteur de concevoir le contexte de l'énonciation comme simple source des proférations étudiées. Le contexte devient une ressource bien plus puissante s'il est également envisagé à la fois comme le lieu de l'émergence des discours récoltés, et comme un giron potentiel de la problématisation du rapport entre l'analyste et son objet d'analyse.

Cette enquête de terrain étaye les observations signalées dans la littérature qui dépeignent l'importance de l'insécurité linguistique chez les sujets sourds, notamment dans leurs productions écrites. L'insécurité linguistique est une attitude du sujet envers sa représentation de ses propres compétences linguistiques et communicationnelles. Elle est constitutivement orientée vers l'autre dans la mesure où cette représentation par le sujet de ses propres compétences émerge par contraste avec sa représentation des compétences de l'allocutaire. C'est contraint par sa représentation de l'allocutaire que le sujet parlant forme l'à dire, le dire, et le dit; et toujours dans une tension entre ses mots à soi et les mots des autres. Le postulat de départ est donc que la matière discursive étudiée est forcément motivée en amont de la profération, en cours de profération, et en relation avec le produit discursif déjà proféré. S'il y a des traces, dans la matière discursive, de motivations (au sens sémantique), et donc d'un potentiel systématique (une régularité dans les atypicités), elles sont certainement plus visibles, plus facilement identifiables en tant que telles, si on les cherche au travers du calque d'une analyse pragmatique conversationnelle préalable, et en s'aidant des balises fournies par un relevé informé des saillances du corpus telles que présentées ici. 


\section{BIBLIOGRAPHIE}

Benveniste, É. (1966). Problèmes de linguistique générale (Gallimard, Vol. 1).

Benveniste, É. (1970). Problèmes de linguistique générale (Gallimard, Vol. 2).

Boutet, D., \& Garcia, B. (2003). Vers une formalisation graphique de la Langue des Signes

Française (LSF) : éléments d'un programme de recherche. Langue Des Signes Française (LSF),

Enjeux Culturels et Pédagogiques, (23), p. 49-62.

Boutet, D., \& Garcia, B. (s.d.). Finalités et enjeux linguistiques d'une formalisation graphique de la Langue des Signes Française (LSF). In: Les langues des signes (LS) : recherches sociolinguistiques et linguistiques. Glottopol, (7), p. 32-52.

Cuxac, C. (2000). La langue des signes française. Les voies de l'iconicité. In: Faits de Langues $15 / 16$, p. 56-57.

Delaporte, Y. (2002). Les sourds, c'est comme ça: ethnologie de la surdimutité. Paris : Maison des sciences de l'homme.

Delaporte, Y. (2007). Dictionnaire étymologique et historique de la langue des signes française: origine et évolution de 1200 signes. Les Essarts-le-Roi : Éditions du Fox.

Détrie, C. (2002). Subjectivité et textualisation. Habilitation à diriger des recherches, Université Paul Valéry Montpellier 3.

Détrie, C., Siblot, P., Verine, B., \& Steuckardt, A. (2017). Termes et concepts pour l'analyse du discours: une approche praxématique (Nouvelle édition augmentée). Paris : Honoré Champion.

Kerbrat-Orecchioni, C. (1996). La Conversation. Paris : Seuil.

Lakoff, G., \& Johnson, M. (2011). Metaphors we live by [with a new afterword] (6. print). Chicago: University of Chicago Press.

Mottez, B. (2006). Les sourds existent-ils ? Paris : L'Harmattan.

Panckhurst, R. et al. (2016). De la collecte à l'analyse d'un corpus de SMS authentiques : une démarche pluridisciplinaire. Histoire Epistémologie Langage, 38, p. 73-85.

Perini, M. (2013). Que peuvent nous apprendre les productions écrites des sourds? Analyse de lectes écrits de personnes sourdes pour une contribution à la didactique du français écrit en formation d'adultes. Université Paris 8, Paris.

Quéré, L. (1999). L’organisation de l'expérience dans un monde sans événement. In P. Gruson \& R. Dulong (éd.), L'Expérience du déni: Bernard Mottez et le monde des sourds en débats. Paris : Éditions de la Maison des sciences de l'homme, 43-60.

Tagg, C. (2012). The discourse of text messaging: analysis of SMS communication. Londres : Continuum.

Wray, A. (2002). Formulaic language and the lexicon. Cambridge; New York: Cambridge University Press. 


\section{NOTES}

1. Toutes les interventions d'anonymisation sont indiquées entre crochets et en majuscules, et tous les extraits du corpus sont retranscrits dans leur orthographe et leur ponctuation d'origine.

2. La requête qui a été faite aux participants de l'enquête de faire don de leurs SMS personnels nous est apparue suffisamment intrusive pour la rendre plus acceptable en évacuant les fichiers transmis dans les fils d'échanges.

3. Pour cette raison, et parce que la plupart des entretiens ont eu lieu dans des endroits publics pour éviter d'alourdir notre intrusion dans la vie personnelle des donneurs, et pour ne pas mettre en place, par mégarde, une situation de domination en les accueillant dans notre domicile ou sur le campus universitaire; nous avons dû faire des choix contraignants dans notre mode de recueil des données et des différentes strates de métadonnées. Nous avons abandonné le projet d'enregistrer les entretiens, et nous en sommes tenus aux notes écrites. La LSF étant une langue visuelle, la discrétion d'un enregistreur audio n'était pas envisageable, et l'encombrement d'un dispositif d'enregistrement vidéo, en plus de contraindre fortement le lieu de rendez-vous (obtenu, somme toute, après de longues tractations), aurait rendu plus difficile l'établissement d'une conversation intrusive et sensible.

4. En plus de faire l'objet de déclarations dans l'entretien que nous avons mené avec elle, ceci est attesté par des diplômes qu'elle a obtenus, ainsi que notre observation de ses pratiques linguistiques dans des cadres professionnels et privés, en français et en LSF.

5. Nous entendons, par l'usage du terme de situation d'énonciation, nous situer dans l'héritage énonciativiste tel qu'il est en usage dès les travaux de Benveniste (Benveniste, 1966a et b), et réactualisé dans les travaux de l'école praxématique de Montpellier (Détrie et al., 2017).

6. ${ }^{6}$ Nous nous situons ici dans l'héritage des analystes de l'interaction.

7. Lors des phases préparatoires du travail de recherche en question, plusieurs interlocuteurs nous ont prévenus que les sourds étaient souvent bien équipés en matière d'informatique. L'explication généralement avancée faisait une grande part à l'attrait des télécommunications pour des personnes qui investissent avec plus d'aisance les médias visuels. Ces commentaires ont souvent été validés par nos observations empiriques sur le terrain, avec une précision importante : si les personnes sourdes que nous avons rencontrées étaient effectivement très bien équipées en matière d'informatique et de télécommunications, il nous a semblé qu'elles n'avaient pas toujours une compétence fine dans les spécificités du maniement de tels dispositifs.

8. La répétition du signe [grêle], proféré avec une mimique signifiant l'intensité de l'épisode climatique, indique la quantité de grêle qui tombe et la densité des chutes de grêlons. Ainsi répété, le signe prend les atours d'un verbe plutôt que d'un substantif. La taille des grêlons est rendue par la configuration des mains, soit fidèlement, soit hyperboliquement.

9. L'indication -parataxe- représente une interruption syntaxique temporaire dans l'activité de signation, et la profération par le locuteur d'une mimique accompagnée d'une variation de posture (et parfois d'une gestuelle para-linguistique, ainsi que d'un regard), qui, ensemble, actualisent un lien logique, et parfois, une modalisation du dire. Dans la transposition de ce tour, la première parataxe actualise la concession, et la seconde actualise la finalité. Il est bien sûr également possible d'employer les signes standards [mais] et [pour], qui existent, mais, en langue des signes proprement dite, on leur préfère souvent les constructions parataxiques. Ces signes standards se rencontrent plus volontiers dans les traductions du français à la LSF, dans les discours effectués en français signé, ou bien dans les boucles métadiscursives où le locuteur cherche à expliciter son propos dans un mouvement analytique, lors de reformulations par exemple.

10. Centre commercial de Montpellier. 
11. On rencontre aussi parfois dans la littérature le mot labième, mais il nous semble entretenir par trop une confusion avec la terminologie phonétique, bien que le terme labialisation ne soit pas totalement satisfaisant sur ce point.

12. On rencontre plus volontiers «espace de signation» dans la littérature, notamment chez $C$. Cuxac, mais nous préférons le terme «volume de signation » qui rend compte plus fidèlement de la réalité corporelle de la profération signée.

13. Une analyse grammaticale du signe [caddie] pris dans le sens de «courses" pointerait une ambigüité sur la nature verbale ou nominale dudit signe pour des raisons morphosyntaxiques qui ne sont pas l'objet du présent travail. Ceci étant posé, il est plus aisé de comprendre la variété des choix de transposition. Nous pourrions très bien dire «je finis les courses pour être plus tranquille ", ou bien " après les courses, je serai plus tranquille »

14. Généralement, en LSF, pour faire d'un substantif un verbe, il suffit souvent de répéter le signe, ou de lui appliquer un mouvement. Certains signes se plient mal à cette transposition cependant.

\section{RÉSUMÉS}

Cet article revient sur un corpus de conversations par SMS entre des personnes sourdes qui présentent un bilinguisme entre le français (écrit) et la Langue des Signes Française (LSF). Ce regard rétrospectif sur ce corpus, notamment sur les motifs de son cadrage, l'enquête de terrain préalable à sa collecte, le protocole de collecte en tant que telle, ou le défi que constitue son annotation, permet de discuter des avantages et des limites des positionnements qualitatifs et quantitatifs en amont de la constitution du corpus.

Le contexte complexe des enjeux culturels, sociaux, économiques, historiques, identitaires, politiques, et épistémologiques, des sourds bilingues devient une ressource bien plus puissante s'il est envisagé à la fois comme le lieu de l'émergence des discours récoltés, et comme un giron potentiel de la problématisation du rapport entre l'analyste et son objet d'analyse.

En décrivant les particularités linguistiques de ce corpus de conversations écrites entre personnes sourdes, cet article cherche à expliciter les écueils analytiques qui peuvent être anticipés ou évités par un travail exploratoire qualitatif tant en amont de la collecte des données et métadonnées, que lors du travail d'analyse.

This article looks back over a corpus of conversational text messages between deaf subjects with (written) French and French Sign Language (FSL) bilingualism. This retrospective overview of this corpus covers in particular its shaping and boundaries, the preliminary fieldwork, the data collection protocol, and the challenges of annotation work. This is an attempt to discuss the benefits and limitations of both quantitative-oriented and qualitative-oriented positioning decisions that need to be made prior to collecting the data.

Deaf bilingualism is deeply wrought by issues of cultural, social, economic, historical, and political nature, as well as identity construction forces and epistemic standpoints. When seen as the locus from which the collected speech emerges, as well as a promising resource for addressing the analyst's relationship to his subject, this complex context proves to be a powerful asset. 
By describing the distinctive linguistic features of this collection of written conversations between deaf subjects, this article aims to elucidate some pitfalls that can be anticipated, prevented, or avoided, with exploratory qualitative fieldwork and analysis protocols.

INDEX

Keywords : corpus collecting, deaf, qualitative analysis, context, bilingualism

Mots-clés : collecte de corpus, surdité, analyse qualitative, contexte, bilinguisme

\section{AUTEUR}

MICHEL OTELL

Praxiling UMR 5267, Université Montpellier III Paul Valéry 